FRI0233

DOES GOUT PROTECT FROM PARKINSON'S DISEASE: A CASE-CONTROL STUDY FROM AN URBAN POPULATION

C. Diaz-Torne ${ }^{1}$, M.A. Pou ${ }^{2}$, F. Orfila ${ }^{3}$, S. Jeria ${ }^{4}$, S. Fernandez ${ }^{4}$, H. Park ${ }^{4}$ I. Castellvi ${ }^{4}$, H. Corominas ${ }^{4} .{ }^{1}$ Unitat de Reumatologia, Hospital de la Santa Creu $i$ Sant Pau; ${ }^{2}$ EAP Encants. ICS; ${ }^{3}$ IDIAP Jordi Gol. ICS; ${ }^{4}$ Unitat de Reumatologia, Hospital de la Santa Creu i Sant Pau, Barcelona, Spain

Background: It has been demonstrated that hyperuricemia protects from Parkinson's disease (PD), but this relation is controversial in patients with gout. Inflammatory properties of urate monosodium(UMS) crystals deposition that appears when uric acid(UA) levels exceed its solubility faces antioxidant and neuroprotective features of soluble UA. Both, UMS crystals deposition and hyperuricemia coexist in gouty patients.

There is several data that demonstrates that hyperuricemia protects from Parkinson's disease (PD). Results from different studies about the relation between them are controversial. Inflammatory properties of the urate crystals faces antioxidant and neuroprotective features of soluble UA. Both, UMS crystals and hyperuricemia coexist in gouty patients.

Objectives: To study if gout protects from Parkinson disease in a mediterranean urban population.

Methods: Primary care based matched case-control study. It has been carried out using the electronic clinical record database from the public health (Institut Català de la Salut) of the city of Barcelona. The database contains anonymous data from almost 1 million and a half people. Just people that were 40 years or older were studied. Were considered cases patients with a PD diagnose, or taking high specific anti-Parkinson's drugs (like I-dope, rasagiline, selegiline...), between 2006 and 2016. For every case we randomly selected four controls matched by gender and age. Data on risk factors was also collected for each individual (diabetes mellitus, hypertension, hypercholesterolemia and tobacco consumption before the PD diagnose), as well as age and gender. A multivariate logistic regression model was used to study the association of gout and PD adjusted by the presence of other risk factors. Adjusted Odds Ratios (aOR) and their $95 \% \mathrm{Cl}$ are provided.

Results: 21789 persons with a PD diagnose and 87156 controls were included. Mean age was 75.5 (SD 10.9) years. 55.6\% were females. 2.5\% of PD patients had a previous gout diagnose, compared to $4.8 \%$ of controls $(p<0.001)$. Multivariate Logistic regression model showed for gout: $\mathrm{aOR}=0.55(0.50-0.61)$. Diabetes mellitus: $\mathrm{aOR}=1.18$ (1.14-1.23); hypertension: $\mathrm{aOR}=0.7(0.72-0.77)$; tobacco: $\mathrm{aOR}=0.55(0.52-0.58)$; high cholesterol levels: $\mathrm{aOR}=0.60(0.58-0.61)$.

Conclusions: Gout shows a protective effect on the development of Parkinson's disease, pointing that the antioxidant and neuro protective effect of UA seems to overcome its inflammatory properties in gouty patients.

Gout shows a protective effect on the development of Parkinson's disease, pointing that the antioxidant and neuro protective effect of UA seems to overcome itsinflammatory properties in gouty patients.

Disclosure of Interest: None declared

DOI: 10.1136/annrheumdis-2018-eular.4666

\section{FRI0234 \\ SEL-212: SELECTIVE MITIGATION OF ANTI-DRUG ANTIBODIES AGAINST PEGSITICASE TO CONTROL SERUM URIC ACID IN HYPERURICEMIC SUBJECTS}

E. Sands ${ }^{1}$, A.J. Kivitz ${ }^{2}$, L. Johnston PhD ${ }^{1}$, W. DeHaan ${ }^{3}$, T.K. Kishimoto'. ${ }^{1}$ Selecta Biosciences, Watertown; ${ }^{2}$ Altoona Center for Clinical Research, Duncansville, $P A$; ${ }^{3} 480$ Arsenal Way, Selecta Biosciences, Watertown, USA

Background: We previously reported initial Phase 2 study results for 60 gout patients treated with SEL-212, a novel combination treatment comprised of pegsiticase (pegylated uricase) co-administered with a synthetic vaccine particle encapsulating rapamycin (SVP-R). ${ }^{1}$ SEL-212 was well-tolerated, mitigated the formation of anti-drug antibodies, and enabled sustained control of serum uric acid (sUA) levels in most subjects. ${ }^{1}$ In this ongoing study, new treatment cohorts involving higher SVP-R dose levels and extended combination regimens were introduced.

Objectives: To assess the tolerability and impact on sUA levels and ADA formation of escalated and expanded doses of SEL-212, designed to be the first nonimmunogenic uricase therapy for refractory gout.

Methods: Patients with symptomatic gout ( $\geq 1$ tophus, gout flare within 6 months or gouty arthropathy) and elevated SUA ( $\geq 6 \mathrm{mg} / \mathrm{dL}$ ) were enrolled to SEL-212 treatment ( $\mathrm{n}=6-12 \mathrm{pts} / \mathrm{cohort}$ ) of fixed doses of pegsiticase $(0.2 \mathrm{mg} / \mathrm{kg}$ or $0.4 \mathrm{mg} /$ $\mathrm{kg}$ ) alone or co-administered with SVP-R $(0.05,0.08,0.1,0.125$, and $0.15 \mathrm{mg} / \mathrm{kg})$. SEL-212 was infused in 28 day cycles $x 3$ doses followed by challenge with pegsiticase alone on 28 day cycles $\times 2$ doses. In addition, 2 cohorts were added that extended the combination treatment with SVP-R to all five q28 doses of pegsiticase. ADAs and sUA were assessed at baseline and 7, 14, 21, and 30 days after each dose to Day 140 . Safety was monitored continuously.

Results: As previously reported, 5 of 6 patients dosed with pegsiticase alone developed ADAs after a single dose, correlating with loss of sUA control by 30 days. The addition of SVP-R to pegsiticase showed a dose-dependent inhibition of ADAs, enabling prolonged control of sUA levels. At a mid-dose of $0.1 \mathrm{mg} / \mathrm{kg}$ SVP-R, combined with either 0.2 or $0.4 \mathrm{mg} / \mathrm{kg}$ pegsiticase, the majority of patients showed sustained control of SUA through 18 weeks. However, control of ADAs and sUA tapered off in some patients by week 20, after two injections of pegsiticase alone. Preliminary results of treatment at increased SVP-R doses $(0.125$ $0.15 \mathrm{mg} / \mathrm{kg}$ ) are consistent with Phase $1 \mathrm{~b}$ data indicating that higher doses of the SVP-R will increase the percentage of patients that remain ADA free. These results also suggest that extending treatment to 5 doses of SVP-R and pegsiticase may enable more subjects to complete the 5 month treatment with sustained reductions in sUA. In addition to mitigating immunogenicity, patients treated with SVP-R experienced a low rate of gout flares, with less than $25 \%$ of patients experiencing flares during the first month after treatment, despite profound drop in sUA level, and continued reduction was observed during months 2-5. SEL-212 has been generally well tolerated at clinically active dose levels and infusion reactions observed with repeat dosing were reduced with increasing doses of SVP-R

Conclusions: SEL-212 has been well tolerated, mitigated immunogenicity, and sustained control of sUA levels. Escalation of the SVP-R component of SEL-212 may enhance the duration of $\mathrm{SUA}$ and $\mathrm{ADA}$ suppression without increased risk.

\section{REFERENCE:}

[1] Sands, et al. EULAR Annual European Congress 2017. Poster THU0402.

Disclosure of Interest: E. Sands Employee of: Selecta Biosciences, A. Kivitz: None declared, L. Johnston PhD Employee of: Selecta Biosciences, W. DeHaan Employee of: Selecta Biosciences, T. Kishimoto Employee of: Selecta Biosciences

DOI: 10.1136/annrheumdis-2018-eular.7396

\section{FRI0235 EFFECT OF SERUM URIC ACID (SUA) ON GOUT FLARES (GF) AND TOPHI RESOLUTION IN GOUT PATIENTS. POOLED POST HOC ANALYSIS OF CLEAR 1 \& CLEAR 2 TRIALS}

F. Perez-Ruiz ${ }^{1}$, P. Richette ${ }^{2}$, R.G. Karra ${ }^{3}$, I. Wild ${ }^{3}$, H. Hagedorn ${ }^{3}$, P. Kandaswamy

${ }^{3}$, T. Bardin ${ }^{2} .{ }^{1}$ Rheumatology Division of Cruces Hospital and University of the Basque Country, Baracaldo, Spain; ${ }^{2}$ Université Paris Diderot, Paris, France; ${ }^{3}$ Grünenthal GmbH, Aachen, Germany

Background: Gout is caused by crystal deposition due to persistent hyperuricemia. EULAR ${ }^{1}$ and other guidelines recommend a target sUA of $<5-6 \mathrm{mg} / \mathrm{dL}(0.30$ to $0.36 \mathrm{mmol} / \mathrm{L}$ ) depending on severity. Despite physiological plausibility and abundant literature evidence there is an absence of consensus among physicians on the targets and necessity of long term urate lowering therapy (ULT) ${ }^{2}$.

Objectives: To investigate the effects of sUA and target sUA $<5-6 \mathrm{mg} / \mathrm{dl}$ with respect to the occurrence of GF, flares requiring treatment (GFRT) and the percent change in the area of tophi (TR) from baseline irrespective of treatment arms. Methods: Data of 1213 patients (PT) was pooled from CLEAR trials ${ }^{384}$, two randomised, double-blind, placebo-controlled Phase 3 studies that evaluated Lesinurad $200 / 400 \mathrm{mg}$ daily in combination with allopurinol vs allopurinol. Prophylaxis for flares was given from baseline to month (M) 5. PT who met the sUA target of $<5-6 \mathrm{mg} / \mathrm{dl}$ at $\mathrm{M} 6$ and 12 were compared against those not on target with respect to the TR from baseline using a Wilcoxon test. In addition, the mean sUA measurements for PT with or without GFs and GFRT was compared at M 6 and 12 using a t-test.

Results: PT with sUA on target $<5-6 \mathrm{mg} / \mathrm{dl}$ showed a larger decrease (increase in percent reduction) in area of tophi with a difference of $27.9 \%$, and $17.3 \%$ at $\mathrm{M}$ 12 only compared to the PT not on target (table 1). The mean sUA levels were $0.227 \mathrm{mg} / \mathrm{dL}$ lower for PT with GF and $0.389 \mathrm{mg} / \mathrm{dL}$ lower for PT with GFRT compared to PT without GF and GFRT at M 6 (table 2), but not at M 12.

Abstract FRI0235 - Table 1. Percent Change from baseline Area of tophi vs. SUA on target $<5-6 \mathrm{mg} / \mathrm{dL}$ at $\mathrm{M} 6$ and 12

\begin{tabular}{|c|c|c|c|c|}
\hline $\begin{array}{l}\text { sUA }(\mathrm{mg} / \mathrm{dL}) \\
\mathrm{n}=\mathrm{M} 6 / \mathrm{M} 12\end{array}$ & $\begin{array}{c}\text { M6 } \\
\text { Median (Q1, Q3) }\end{array}$ & p-value\# & $\begin{array}{c}\text { M12 } \\
\text { Median (Q1, Q3) }\end{array}$ & p-value\# \\
\hline $\begin{array}{l}<5(n=31 / 36) \\
>5(n=93 / 87)\end{array}$ & $\begin{array}{l}-22.1(-46.4,5.63) \\
-22-2(-56.7,6.91)\end{array}$ & 0.78 & $\begin{array}{l}-59.6(-98.3,-27.5) \\
-31.7(-77.4,13.06)\end{array}$ & 0.01 \\
\hline $\begin{array}{l}<6(n=63 / 66) \\
\geq 6(n=61 / 57)\end{array}$ & $\begin{array}{c}-23.8(-56.7,1.59) \\
-19.2(-42.5,10.30)\end{array}$ & 0.64 & $\begin{array}{l}-49.0(-93.3,-12.9) \\
-31.7(-60.1,13.06)\end{array}$ & 0.06 \\
\hline
\end{tabular}


$\S$ Number of PT with a target tophus at baseline was small. \# Wilcoxon test with H0: Mean ranks in categories are equal

Abstract FRI0235 - Table 2. Mean sUA at M 6 and 12 by occurrence of GF and GFRT

\begin{tabular}{lcccc}
\hline Mean sUA mg/dL $(\mathbf{9 5} \% \mathbf{~ C l})$ M6 & p-value\# & Mean sUA $(\mathbf{9 5} \%$ Cl) M12 & p-value\# \\
\hline GF Yes & $5.94(5.80-6.07)$ & & $5.92(5.79-6.06)$ & \\
\hline GF No & $5.71(5.51-5.92)$ & & $5.91(5.64-6.17)$ & \\
Difference & $0.22(-0.01-0.47)$ & 0.06 & $0.01(-0.28-0.31)$ & 0.91 \\
GFRT No & $5.91(5.79-6.03)$ & & $5.93(5.80-6.05)$ & \\
GFRT Yes & $5.530(5.23-5.83)$ & & $5.83(5,41-6.25)$ & \\
Difference & $0.38(0.06-0.71)$ & 0.01 & $0.09(-0.34-0.53)$ & 0.65 \\
\hline
\end{tabular}

\# T-test with $\mathrm{HO}$ : No difference in means

Conclusions: These results confirm that sUA on target $<5-6 \mathrm{mg} / \mathrm{dl}$ is essential for TR, and longer the control better the TR. The lower mean sUA levels for PT with GF and GFRT was observed at M6 and not at M 12, maybe owing to the fact that flares are common during the first months of ULT initiation and then taper off and urate deposits were more fragile and not completely dissolved. Also M 6 and 12 may not be optimal to observe statistically significant differences between treatments with respect to TR, GF and GFRT.

\section{REFERENCES:}

[1] Richette $P$, et al. Ann Rheum Dis 2017;76:29-42

[2] Qaseem A, et al. Ann Intern Med 2017;166(1):58-68.

[3] Saag KG, et al. Arthritis Rheumatol 2017;69:203-12.

[4] Bardin T, et al. Ann Rheum Dis 2017;76:811-20.

Disclosure of Interest: F. Perez-Ruiz Consultant for: Grünenthal, Menarini, Speakers bureau: Grünenthal, Menarini, P. Richette Consultant for: Grünenthal, Speakers bureau: Grünenthal, R. Karra Employee of: Grünenthal, I. Wild Employee of: Grünenthal, H. Hagedorn Employee of: Grünenthal, P. Kandaswamy Employee of: Grünenthal, T. Bardin Consultant for: Grünenthal DOI: 10.1136/annrheumdis-2018-eular.5565

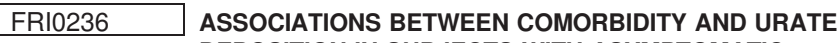 DEPOSITION IN SUBJECTS WITH ASYMPTOMATIC HYPERURICEMIA: A PILOT STUDY}

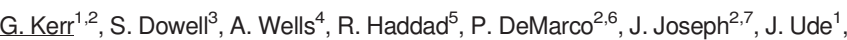
S. Hochberg ${ }^{1}$, J. Huang ${ }^{8}$, D. Nashel ${ }^{2,7} .{ }^{1}$ VAMC/HUH; ${ }^{2}$ Georgetown University Hospital; ${ }^{3}$ Howard University, Washington; ${ }^{4}$ Rheumatology/Immunotherapy, Franklin; ${ }^{5}$ The Hospital for Special Surgery, New York; ${ }^{6}$ Arthritis and Rheumatism Associates, PC, Wheaton; ${ }^{7}$ VAMC; ${ }^{8}$ MedStar Health Research Institute,

Washington, USA

Background: Hyperuricemia is common and along with comorbidities, is increasing in prevalence. Though often asymptomatic and hence, under diagnosed, it may be associated with subclinical urate deposition. Ultrasound (US) imaging can detect urate deposition in individuals with asymptomatic hyperuricemia (ASU). Objectives: To evaluate the association of comorbidities with urate deposition via US in ASU.

Methods: ASU was defined as serum urate (sUA) $>6 \mathrm{mg} / \mathrm{dl}$; sUA $<6 \mathrm{mg} / \mathrm{dl}$ served as controls. Demographic (age, gender, BMI), comorbidity (CM - [hypertension (HTN), hyperlipidemia (HLD), diabetes mellitus (DM), cardiovascular (CVD) and renal disease (CKD)], diuretic/aspirin use, dietary data (alcohol, red meat, seafood) were collected. Ultrasonography (US) of joints (knee/MTP), tendons (triceps, quadriceps/patella, Achilles) was performed via standard procedure, OMERACT parameters of urate deposition documented, and images read by an Expert ultrasonographer blinded to sUA category. Correlations between sUA levels and MSK urate deposition with comorbidities, medication and dietary risk factors were analysed by 2 -stage multivariable logistic regression model with propensity score weighting.

Results: Of 95 predominantly non-Hispanic Blacks (mean age 59.7 years, BMI $\left.\sim 32 \mathrm{~kg} / \mathrm{m}^{2}\right)$, ASU subjects $(n=71$, median $\mathrm{sUA}=8.0$ ) were older men, with more frequent HTN, CVD, CKD, alcohol ingestion versus controls. In multivariate analyses adjusting for demographic characteristics, BMI, CKD, and alcohol use were positively associated with sUA >6 mg/dl; while HTN, CVD, and CKD were positively associated with sUA >8 mg/dl. Adjusting for comorbidities, sUA >8 mg/dl was significantly associated with urate deposition at knee ( $O R=3.20 ; p=0.03)$, quadriceps and Achilles tendons $(O R=4.14 ; p<0.01), O R=9.51 ; p<0.01$, respectively) but not at 1 st MTP $(O R=2.14 ; p=0.06)$. A sUA $>6 \mathrm{mg} / \mathrm{dl}$ alone, however, did not predict urate deposition.

Conclusions: Presence of HTN, CVD and CKD are associated with higher levels of SUA and increases the risk of urate deposition in ASU patients. Identifying a subset of ASU patients that may benefit from urate lowering therapy requires further stratification and long term follow up for incident gouty arthritis in order to alter current urate lowering treatment guidelines.

Disclosure of Interest: G. Kerr Grant/research support from: Ardea, Grunenthal Horizon, S. Dowell Grant/research support from: Horizon, A. Wells: None declared, R. Haddad: None declared, P. DeMarco: None declared, J. Joseph: None declared, J. Ude: None declared, S. Hochberg: None declared, J. Huang: None declared, D. Nashel: None declared DOI: 10.1136/annrheumdis-2018-eular.2959

\section{FRI0237 PEGLOTICASE TREATMENT SIGNIFICANTLY DECREASES MEAN ARTERIAL BLOOD PRESSURE IN PATIENTS WITH CHRONIC GOUT}

H. Choi ${ }^{1}$, R. Johnson ${ }^{2}$, A. Yeo ${ }^{3}$, P. Lipsky ${ }^{4} .{ }^{1}$ Massachusetts General Hospital, Boston; ${ }^{2}$ University of Colorado, Aurora; ${ }^{3}$ Horizon Pharma, Lake Forest; ${ }^{4}$ AMPEL BioSolutions LLC, Charlottesville, USA

Background: There are significant correlations between serum uric acid (sUA) and blood pressure (BP) in individuals with and without gout. ${ }^{1}$ Limited data sug gest that lowering sUA may decrease $\mathrm{BP}^{2}$, but no consistent effect has been noted. ${ }^{3}$ Recent guidelines suggest the need for more aggressive management of increased BP. 4

Objectives: To determine the impact of persistent, very low sUA levels on BP in subjects with chronic refractory gout treated with pegloticase, a recombinant uricase conjugated to polyethylene glycol approved for chronic refractory gout that decreases sUA to $<1 \mathrm{mg} / \mathrm{dL}$.

Methods: This analysis used results from two, 6 month randomised clinical trials (RCTs) in which subjects were treated with $8 \mathrm{mg}$ pegloticase every 2 or 4 weeks (q2w or q4w) or placebo. ${ }^{5}$ sUA responders maintained $\mathrm{sUA}<6 \mathrm{mg} / \mathrm{dL} .{ }^{5}$ Sitting BP was measured at each visit and estimated glomerular filtration rate (eGFR) was determined at baseline and after 3 and 6 months

Results: Serial BP measures were obtained in 173 subjects during the course of the RCTs. Significant reductions in mean arterial pressure (MAP) from baseline to 6 months were noted in $q 2 w$ responders $(p=0.0029)$ (figure 1$)$, whereas reductions in MAP in other groups were not significant. Notably, 18/29 (62.1\%) of q2w sUA responders experienced persistent reductions in MAP ( $p=0.01$ compared to other groups). Changes in both systolic and diastolic BP paralleled the change in MAP. Of the q2W sUA responders exhibiting persistent decreases in MAP, there were no significant differences in baseline age, gender, race, body mass index history of hypertension, gout duration, MAP, sUA, cholesterol, eGFR, or urinary UA/creatine ratio compared with those who did not lower MAP. There were no significant changes in eGFR in SUA responders to pegloticase treatment over the course of the study and there was no significant correlation between change from baseline MAP and eGFR in these subjects $(p=0.43)$.

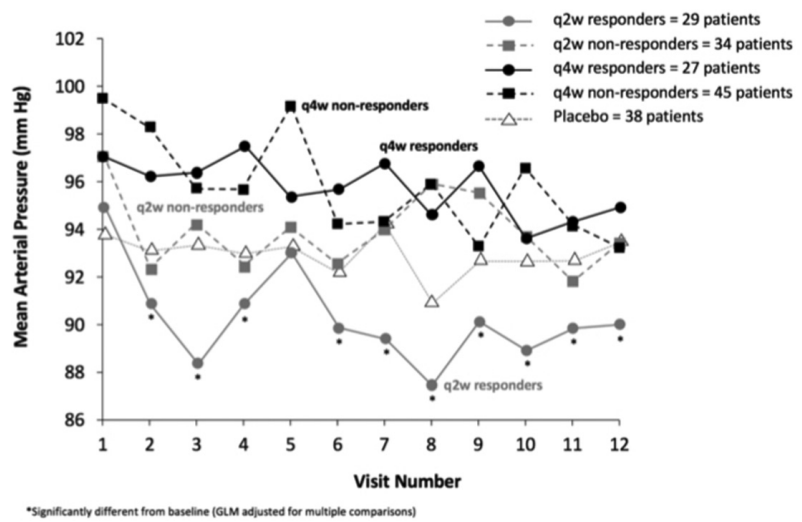

Abstract FRI0237 - Figure 1. *Significantly different from baseline (GLM adjusted for multiple comparisons)

Conclusions: Responders to biweekly pegloticase experienced significant reductions in MAP that were independent of changes in renal function.

\section{REFERENCES:}

[1] Lee JJ, et al. J Clin Hyperten 2013;15:435

[2] Agarwal V, et al. J Clin Hyperten 2013;15:435

[3] Franca GPH, et al. Cochrane Database Syst Rev. 2017;4:CD008652.

[4] Whelton PK, et al. J Am Coll Cardiol 2017 Nov 13. [Epub ahead of print]

[5] Sundy JS, et al. JAMA 2011;306:711. 